\title{
Alterations in BDNF Protein Concentrations in the Hippocampus do not Explain the Pro-neurogenic Effect of Citalopram on Adult Neurogenesis
}

\section{Running title: The interplay of serotonin and BDNF in antidepressant action}

Markus Petermann ${ }^{1,2 \$}$, Golo Kronenberg ${ }^{3,4 \$}$, Valentina Mosienko ${ }^{1,7}$, Michael Bader ${ }^{1,2,5,6,7}$, Natalia Alenina $^{1,6,8}$, Rainer Hellweg ${ }^{4 *}$, Friederike Klempin ${ }^{1,2,4 * \#}$

${ }^{1}$ Max Delbrück Center for Molecular Medicine (MDC), 13125 Berlin, Germany

2Berlin Institute of Health, 10178 Berlin, Germany

3 University of Leicester and Leicestershire Partnership NHS Trust, Leicester LE3 9Q4, UK

${ }^{4}$ Department of Psychiatry and Psychotherapy, Charité - University Medicine Berlin, 10117 Berlin, Germany

${ }^{5}$ Charité - University Medicine Berlin, Berlin, Germany

${ }^{6}$ DZHK (German Centre for Cardiovascular Research), partner site Berlin, Germany

7University of Lübeck, 23562 Lübeck, Germany

${ }^{8}$ Institute of Cytology, Russian Academy of Science, St. Petersburg, Russia

${ }^{9}$ current address: University of Exeter, College of Medicine and Health, EX4 4PS, Exeter, UK

\$, *These authors contributed equally

\#Corresponding author: Friederike Klempin

Max Delbrueck Center

13125 Berlin, Germany

Email: friederike.klempin@mdc-berlin.de

ORCID Friederike Klempin: 0000-0003-3171-0497

\section{Keywords}

antidepressants, BDNF, tryptophan hydroxylase 2 (TPH2), citalopram, neurogenesis

\section{Conflict of interest}

The authors have no conflicts of interest to declare. 


\section{Abstract}

Introduction Brain-derived neurotrophic factor (BDNF) has been implicated in the pro-neurogenic effect of selective serotonin reuptake inhibitors. In this study, we used Tph2 ${ }^{--}$mice lacking brain serotonin to dissect the interplay between BDNF and the serotonin system in mediating the effects of antidepressant pharmacotherapy on adult neurogenesis in the hippocampus.

Methods Besides Citalopram (CIT), we tested Tianeptine (TIA), an antidepressant whose mechanism of action is not well understood. Specifically, we examined cell survival and endogenous concentrations of BDNF following daily injection of the drugs.

Results 21 days of CIT, but not of TIA, led to a significant increase in the survival of newly generated cells in the dentate gyrus of wild-type mice, without a significant effect on BDNF protein levels by either treatment. In Tph2-- mice, adult neurogenesis was consistently increased. Furthermore, Tph2-- mice showed increased BDNF protein levels, which were not affected by TIA but significantly reduced by CIT.

Discussion We conclude that the effects of CIT on adult neurogenesis are not explained by changes in BDNF protein concentrations in the hippocampus.

\section{Introduction}

Selective serotonin reuptake inhibitors (SSRIs) increase serotonin neurotransmission. Citalopram (CIT) is a commonly prescribed SSRI that swiftly increases the synaptic availability of serotonin by inhibiting the serotonin transporter [Rev in [1]]. Tianeptine (TIA), an antidepressant whose mechanism of action remains to be clearly defined, paradoxically appears to act as a selective serotonin reuptake enhancer (SSRE) [2]. However, recent evidence suggests that modulation of synaptic glutamate transmission in limbic structures maybe more important to TIA's antidepressant effects \{Rev in [3]]. Chronic antidepressant treatment is required for alleviating depression. Moreover, a fairly large number of patients do not respond satisfactorily to pharmacotherapy. A wealth of evidence has demonstrated adaptive neurobiological responses to chronic SSRI treatment. These include a delayed increase in neurogenesis in the adult hippocampus in rodents [4, 5] alongside altered brain-derived neurotrophic factor (BDNF) signaling [6-8].

So far, it has not been clearly established if, and how, BDNF is involved in mediating the pro-neurogenic effect of antidepressants targeting the serotonin system. Here, we address this question by studying antidepressant effects on BDNF protein levels and neurogenesis in the hippocampus of wild-type control (CTR) and tryptophan hydroxylase 2 deficient ( Tph2--) mice selectively depleted of brain serotonin [9]. Tph2 - mice have been characterized in detail earlier [10, 11]. Briefly, these mice exhibit transient growth retardation, aggressive behaviors, and increased despair-like responses [11, 12]. This combination of symptoms mirrors important aspects of the clinical presentation of depressed patients with reduced central serotonin function (i.e., low 5-hydroxyindole acetic acid [5-HIAA] levels in cerebrospinal fluid [13, 14]). Using Tph2 ${ }^{--}$animals, we and others demonstrated that depletion of brain serotonin increases BDNF expression 
in hippocampus and prefrontal cortex [15-17]; and that serotonin is required for the biological effects of electroconvulsive therapy [18]. In this study, Tph2-- and CTR mice were treated with CIT or TIA for 21 days. We studied the survival and phenotype of newly generated cells in tandem with BDNF protein levels in hippocampus.

\section{Methods}

Animals and treatments. The work presented here is part of an extended project aimed at studying the neurobiology of antidepressant treatments using Tph2-- mice $[10,16,18]$. Tph2 $\%$ mice [9] were backcrossed to a C57BL/6N background for more than 10 generations. All mice were housed four to six per cage (floor space $530 \mathrm{~cm}^{2}$, Tecniplast, Germany) under standard laboratory conditions with a light/dark cycle of 12 hours and ad libitum access to food and water. In line with local requirements for animal keeping, cages were fitted with a Mouse Igloo® (Zoon lab, Germany). Eight-week-old female Tph2 ${ }^{-1}$ and CTR mice $(\mathrm{n}=38)$ were randomly assigned to daily intraperitoneal (i.p.) injections of: 1) SAL (0.9\% saline), 2) CIT (Citalopram hydrobromide, $10 \mathrm{mg} / \mathrm{kg}$ body weight, dissolved in 0.9\% saline; Lundbeck, Hamburg, Germany), or 3) TIA (Tianeptine sodium salt, $10 \mathrm{mg} / \mathrm{kg}$ body weight dissolved in $0.9 \%$ saline; Tocris Bioscience, Germany). Injections were performed shortly before the start of the active phase of the animals. Dosages were chosen based on prior reports in the literature (e.g. $[8,19,20])$. Mice received three i.p. injections, 6 hours apart, of BrdU (5-bromo-2'-deoxyuridine, 50 mg/kg; Sigma-Aldrich, Germany) on day 0, followed by daily drug treatment for 21 days (Fig. 1A). Mice were killed on the following morning (day 22) between 9 and 11 am. To determine BDNF protein levels, another cohort of mice $(n=35)$ was sacrificed on day 22 following the same treatments, and hippocampi of both hemispheres were micro-dissected. All procedures had been approved by the local animal welfare and ethical review body and were carried out in accordance with the European Communities Council Directive 2010/63/UE.

Tissue preparation, immunohistochemistry, and BDNF quantification. Mice were deeply anesthetized and perfused transcardially with $0.9 \%$ saline. Brains were removed and placed into $4 \%$ paraformaldehyde for immunohistochemistry, while micro-dissected hippocampi for BDNF analysis were snap-frozen. Endogenous levels of BDNF were measured in the thawed homogenates using a commercial enzymelinked immunosorbent assay (Promega, United States). As described in detail previously, the manufacturer's instructions were adapted to a highly sensitive fluorometric technique [21]. BrdU immunohistochemistry followed the peroxidase method in accordance with an established protocol [10]. Briefly, one-in-six series of sequential $40 \mu \mathrm{m}$ coronal brain sections were stained, and immunoreactive cells were counted throughout the rostro-caudal extent of the dentate gyrus. The total number of BrdU-positive cells was estimated by multiplying cell counts by six. For immunofluorescence, the following primary antibodies were applied: anti-BrdU (rat, 1:500; Bio-Rad AbD Serotec, Germany), anti-Calretinin (rabbit, 1:1000; Sigma-Aldrich, Germany), anti-doublecortin (DCX; goat, 1:250; Santa Cruz Biotechnology, United States), anti-NeuN (rabbit, 1:200; Abcam, United Kingdom), and anti-Sox2 (goat, 1:1000; Santa Cruz 
Biotechnology, United States). Secondary antibodies conjugated to Alexa488, Cy3, and Cy5 were used (1:250, Invitrogen, Germany). Approximately 50 BrdU-positive cells per dentate gyrus were randomly selected for phenotypic analysis using a Leica TCS SP5 confocal microscope (Leica, Germany).

Statistical analysis. Two-way ANOVA was followed by Tukey's post hoc test where appropriate (PRISM software). Student's $t$ test was used for individual pairwise comparisons. For phenotypic analyses, two-way ANOVA was performed for each marker individually. All values are expressed as mean \pm SEM. $P$ values of $\leq 0.05$ were considered statistically significant.

\section{Results}

\section{Tph2 $^{--}$phenotype reflects treatment effect of CIT on adult neurogenesis}

Mice were given three injections of $\mathrm{BrdU}$ at 6-hour intervals on day 1. Animals then received daily i.p. injections of CIT, TIA, or saline for 21 days (Fig. 1A). We assessed the survival of newly generated cells in the dentate gyrus (Fig. 1B; $F(2,32)=4.0, p_{\text {interaction }}=0.029 ; F(2,32)=0.99, p$ treatment $=0.38 ; F(1,32)=18.0, p$ genotype $=0.0002)$. CIT treatment increased the survival of BrdU-positive cells in CTR mice (SAL $261 \pm 34$ vs. CIT $408 \pm 50$ cells, $p=0.035$; Fig. 1B) while treatment with TIA had no effect ( $204 \pm 21$ cells, $p=0.176$; Fig. 1B). BrdU numbers in Tph2 $\%$ mice were significantly elevated relative to CTR mice in the SAL and TIA conditions without significant differences between treatment groups (Fig. 1B). Taken together, CIT treatment does not promote the survival of newly generated cells in the absence of brain serotonin, with TPH2 deficiency recapitulating the effects of CIT on adult neurogenesis observed in CTR mice.

\section{CIT treatment decreases BDNF protein levels in Tph2/- mice}

Next, we measured BDNF protein levels in the dissected hippocampi of Tph2 $\%$ and CTR mice at 21 days. In CTR mice, BDNF signaling was not affected by treatment with either CIT or TIA (Fig. 1C). However, a treatment and genotype effect was observed in Tph2/- mice $\left(F(2,29)=7.59, p_{\text {treatment }}=0.002 ; F(1,29)=9.90\right.$, $p_{\text {genotype }}=0.004$; Fig. $1 \mathrm{C}$ ). As compared to CTR mice, BDNF protein levels in Tph2 ${ }^{-}$mice were significantly increased in the SAL (75\%) and TIA (69\%) conditions, while BDNF levels following CIT decreased significantly in Tph2 ${ }^{-1}$ mice (Tukey's post hoc test: $p=0.001$; Fig. 1C) such that differences between genotypes were no longer detected. These results indicate that the effect of CIT treatment on adult neurogenesis observed in CTR is not mediated by changes in BDNF protein level. Furthermore, in the absence of brain serotonin, CIT reduces BDNF concentrations in the hippocampus.

\section{Tph2/- phenotype reflects treatment effect of CIT on developmental milestones of new neurons}

BrdU-positive cells were investigated for co-expression of Sox2 (precursor cells), DCX (transient immature neurons), Calretinin (CR, transient marker of early post-mitotic neurons), and NeuN (mature granule cells; Fig. 1D). Phenotypic analysis revealed an increase in the number of BrdU/Sox2, BrdU/DCX, and 
BrdU/NeuN-expressing cells in CTR mice following CIT treatment (Fig. 1E; Sox2, $F(1,17)=10.98, p_{\text {genotype }}=$ 0.004 ; DCX, $\mathrm{F}(2,16)=9.40, p_{\text {treatment }}=0.002, \mathrm{~F}(1,16)=5.39, p_{\text {genotype }}=0.034 ; \mathrm{NeuN}, \mathrm{F}(2,16)=3.60, p_{\text {treatment }}$ $=0.05$ ). Next, we studied the relative distribution of markers within the neuronal lineage in CTR mice (Fig. $1 F)$. We observed a significant decrease in BrdU/CR cells $\left(C R, F(2,17)=4.21, p_{\text {treatment }}=0.033\right)$ in tandem with an increase in the number of NeuN-immunoreactive BrdU cells in response to $\mathrm{CIT}$ ( $p=0.045$, Fig. 1F). Surprisingly, although TIA treatment did not affect the absolute number of BrdU-positive cells, BrdU/DCX cells in CTR mice treated with TIA were significantly decreased both in terms of absolute numbers $(p=$ 0.002; Fig. 1E) and relative phenotypic distribution $(\mathrm{F}(2,16)=9.99, p=0.002$; Fig. $1 \mathrm{~F})$.

The Tph2 $\%$ phenotype again recapitulated the effects of CIT in CTR mice. The number of BrdU/Sox2, BrdU/DCX, BrdU/CR, and BrdU/NeuN-positive cells in Tph2\%-mice and in CTR CIT mice was comparable (Fig. 1E). Independent of treatment condition, the proportion of BrdU/CR in all BrdU-positive cells was similar to that seen in CTR CIT mice $\left(\mathrm{CR}, \mathrm{F}(1,17)=14.27, p_{\text {genotype }}=0.0015\right.$; Tukey's post hoc tests, SAL $p$ $=0.050$, TIA $p=0.003$; Fig. 1F). Overall, numbers and relative phenotypic distributions of any marker were relatively unaffected by treatment condition in Tph2 $\%$ mice. CIT treatment slightly increased the proportion of BrdU/DCX cells relative to SAL ( $p=0.048$; Fig. $1 \mathrm{~F})$.

\section{Discussion}

Our results suggest that the effects of CIT on BDNF protein concentrations in the hippocampus are largely separable from CIT effects on neurogenesis in the dentate gyrus. Chronic CIT over a treatment period of 21 days enhanced adult neurogenesis in CTR mice without affecting BDNF protein levels. Furthermore, in the absence of brain serotonin, CIT strongly decreased BDNF concentrations while the survival of newborn cells remained unchanged.

The findings presented here build on our earlier experiments studying BDNF in brain tissue of Tph2 ${ }^{--}$mice. We replicate our previous observation of increased BDNF protein concentrations in the hippocampus of mice developmentally depleted of brain serotonin [16]. We speculate that increased BDNF concentrations represent a compensatory mechanism in the absence of serotonin. A different mouse model, Tph2::eGFP knock-in mice, similarly showed enhanced BDNF mRNA alongside hyperinnervation of serotonin fibers in the hippocampus. The finding that chronic CIT did not elevate BDNF concentrations in the hippocampus is in keeping with an earlier study in rats which likewise did not detect increased BDNF protein levels following chronic antidepressant treatment with either escitalopram or desipramine [22]. We here expand on this finding by demonstrating, in parallel experiments in mice, that chronic CIT increases neurogenesis but leaves BDNF levels unaffected. Notably, we did not investigate components of the BDNF pathway such as tyrosine kinase receptor $B$ or the p75 neurotrophin receptor. Furthermore, the antibody to detect BDNF used in this study reacts with both mature BDNF and its precursor pro-BDNF. Therefore, our findings do not 
represent the full picture of BDNF signaling [23]. Further points should be mentioned. Based on our earlier study in these mice [16], BDNF protein levels tend to be higher in female than in male animals and we therefore chose to limit the current investigation to female mice. Future studies may explore the effects of sex on BDNF levels further in these animals. A separate, but closely related, point is that of housing conditions. Living in an enriched environment has been shown to increase BDNF protein in the hippocampus specifically in male $\mathrm{BDNF}^{+/-}$mice [24]. It should therefore also be stressed that the mice investigated here were kept under standard housing conditions.

The finding that TIA affects neither neurogenesis nor BDNF is somewhat unexpected, but may possibly be due to the fact that the mechanism of action of TIA differs significantly from that of other antidepressants. However, it must not go unmentioned that an earlier study in adult male tree shrews did not find an effect of long-term TIA on cell proliferation in the dentate gyrus, which is in line with our results [25]. Instead, the neurobiological responses to TIA involve reversal of stress-induced impairments in glutamate neurotransmission [3].

The genetic approach used herein also allowed us to dissect serotonin-dependent and serotoninindependent drug effects. The fact that the high BrdU numbers found in Tph2/- mice were similar across the three treatment conditions (no difference to SAL control, 'between group effect') suggests that the proneurogenic effects of CIT require serotonin signaling in the brain $[4,19]$. It is interesting to note that the increase in the number of BrdU cells detected in Tph2 - mice relative to CTR mice is consistent with similar findings of increased neurogenesis in two other murine models of constitutive serotonin depletion (i.e., Pet1/ and VMAT2SERT-Cre mice; [26]). It remains to be elucidated which mechanisms lead to increased neurogenesis under these opposite conditions, of either absence of serotonin from the adult brain or increased serotonin in the synaptic cleft. Importantly, the fact that the increase in neurogenesis under these experimental conditions was comparable does not necessarily indicate that the underlying biological processes are the same. Finally, we found that CIT strongly suppressed BDNF expression in Tph2- mice. At least two explanations of this unexpected finding are possible: i) second-order effects of CIT mediated by direct actions on peripheral serotonin (e.g., in platelets), which is not affected by TPH2 deficiency. This hypothesis deserves to be investigated further using mice lacking both, the peripheral (TPH1) and the central (TPH2) isoform; ii) effects of the CIT molecule that are independent of its action as an SSRI. This hypothesis could be explored by testing the effects of other SSRIs such as sertraline and paroxetine in Tph2-- mice. Another logical extension of this work would be to determine the effects of CIT in BDNF+/- mice, which, remarkably, have been shown to exhibit increased extracellular serotonin levels in hippocampus [27].

The results reported herein should be briefly placed in the broader context of serotonin-neurotrophin interactions in rodent depression models. Both decreased (BDNF+/- mice) and increased BDNF levels (bilateral BDNF injections) in the hippocampus have been reported to impact serotonin transmission [28]. 
In marked similarity to the finding of increased BDNF levels in the hippocampus of $T p h 2^{-/}$mice, olfactory bulbectomy has previously been shown to result in increased BDNF protein concentrations alongside reduced serotonin turnover [21]. However, bulbectomized mice also display a reduction in hippocampal neurogenesis [29] which was absent in Tph2/ mice. The depression-like phenotype could be reversed by treatment with liver hydrolysate (through activation of phosphate-AMPK) resulting in, alongside an amelioration of the behavioral phenotype, increased BDNF protein levels and increased neurogenesis [29].

To summarize, we here show that the effects of the SSRI CIT on adult neurogenesis and on BDNF protein levels in the hippocampus dissociate. Tph2 ${ }^{-/}$mice display a versatile tool to dissect the neurobiological effects of serotonergic antidepressants. Future studies in conditional knockout models allowing transient serotonin depletion and replenishment may shed further light on the interplay between the serotonin and neurotrophin systems.

\section{References}

1. Sangkuhl K, Klein TE, Altman RB. PharmGKB summary: citalopram pharmacokinetics pathway. Pharmacogenet Genomics 2011; 21(11): 769-72

2. Mennini T, Mocaer E, Garattini S. Tianeptine, a selective enhancer of serotonin uptake in rat brain. Naunyn Schmiedebergs Arch Pharmacol 1987; 336(5): 478-82

3. McEwen BS, Chattarji S, Diamond DM et al. The neurobiological properties of tianeptine (Stablon): from monoamine hypothesis to glutamatergic modulation. Mol Psychiatry 2010; 15(3): 237-49

4. Malberg JE, Eisch AJ, Nestler EJ et al. Chronic antidepressant treatment increases neurogenesis in adult rat hippocampus. J Neurosci 2000; 20(24): 9104-10

5. Santarelli L, Saxe M, Gross C et al. Requirement of hippocampal neurogenesis for the behavioral effects of antidepressants. Science 2003; 301(5634): 805-9

6. Molendijk ML, Bus BA, Spinhoven P et al. Serum levels of brain-derived neurotrophic factor in major depressive disorder: state-trait issues, clinical features and pharmacological treatment. Mol Psychiatry $2011 ; 16(11): 1088-95$

7. Nibuya M, Nestler EJ, Duman RS. Chronic antidepressant administration increases the expression of cAMP response element binding protein (CREB) in rat hippocampus. J Neurosci 1996; 16(7): 2365-72

8. Russo-Neustadt AA, Alejandre H, Garcia $\mathrm{C}$ et al. Hippocampal brain-derived neurotrophic factor expression following treatment with reboxetine, citalopram, and physical exercise. Neuropsychopharmacology 2004; 29(12): 2189-99

9. Alenina N, Kikic D, Todiras $\mathrm{M}$ et al. Growth retardation and altered autonomic control in mice lacking brain serotonin. Proc Natl Acad Sci U S A 2009; 106(25): 10332-7

10. Klempin $F$, Beis $D$, Mosienko $V$ et al. Serotonin is required for exercise-induced adult hippocampal neurogenesis. J Neurosci 2013; 33(19): 8270-5

11. Mosienko V, Bert B, Beis $\mathrm{D}$ et al. Exaggerated aggression and decreased anxiety in mice deficient in brain serotonin. Transl Psychiatry 2012; 2: e122

12. Gutknecht L, Popp S, Waider J et al. Interaction of brain 5-HT synthesis deficiency, chronic stress and sex differentially impact emotional behavior in Tph2 knockout mice. Psychopharmacology (Berl) 2015; 232(14): 2429-41

13. Mann JJ, Malone KM. Cerebrospinal fluid amines and higher-lethality suicide attempts in depressed inpatients. Biological Psychiatry 1997; 41(2): 162-71

14. Placidi A, Sartori S. Fine needle sampling cytology of palpable breast lesions with immediate reporting. Acta Cytol 2001; 45(5): 894-6

15. Brivio P, Sbrini G, Peeva P et al. TPH2 Deficiency Influences Neuroplastic Mechanisms and Alters the Response to an Acute Stress in a Sex Specific Manner. Front Mol Neurosci 2018; 11: 389 
16. Kronenberg G, Mosienko V, Gertz K et al. Increased brain-derived neurotrophic factor (BDNF) protein concentrations in mice lacking brain serotonin. Eur Arch Psychiatry Clin Neurosci 2015:

17. Migliarini S, Pacini G, Pelosi B et al. Lack of brain serotonin affects postnatal development and serotonergic neuronal circuitry formation. Mol Psychiatry 2012:

18. Kronenberg G, Petermann M, Dormann $C$ et al. Brain serotonin critically contributes to the biological effects of electroconvulsive seizures. Eur Arch Psychiatry Clin Neurosci 2018; 268(8): 861-4

19. Klempin F, Babu H, De Pietri Tonelli D et al. Oppositional effects of serotonin receptors 5-HT1a, 2, and $2 \mathrm{c}$ in the regulation of adult hippocampal neurogenesis. Front Mol Neurosci 2010; 3:

20. Mombereau C, Gur TL, Onksen J et al. Differential effects of acute and repeated citalopram in mouse models of anxiety and depression. Int J Neuropsychopharmacol 2010; 13(3): 321-34

21. Hellweg R, Zueger M, Fink $\mathrm{K}$ et al. Olfactory bulbectomy in mice leads to increased BDNF levels and decreased serotonin turnover in depression-related brain areas. Neurobiol Dis 2007; 25(1): 1-7

22. Jacobsen JP, Mørk A. The effect of escitalopram, desipramine, electroconvulsive seizures and lithium on brain-derived neurotrophic factor $\mathrm{mRNA}$ and protein expression in the rat brain and the correlation to 5-HT and 5-HIAA levels. Brain Res 2004; 1024(1-2): 183-92

23. Polacchini A, Metelli G, Francavilla R et al. A method for reproducible measurements of serum BDNF: comparison of the performance of six commercial assays. Sci Rep 2015; 5: 17989

24. Chourbaji S, Hörtnagl H, Molteni R et al. The impact of environmental enrichment on sex-specific neurochemical circuitries - effects on brain-derived neurotrophic factor and the serotonergic system. Neuroscience 2012; 220: 267-76

25. Czeh B, Michaelis T, Watanabe T et al. Stress-induced changes in cerebral metabolites, hippocampal volume, and cell proliferation are prevented by antidepressant treatment with tianeptine. Proc Natl Acad Sci U S A 2001; 98(22): 12796-801

26. Diaz SL, Narboux-Neme N, Trowbridge $S$ et al. Paradoxical increase in survival of newborn neurons in the dentate gyrus of mice with constitutive depletion of serotonin. Eur J Neurosci 2013; 38(5): 2650-8

27. Guiard BP, David DJ, Deltheil $T$ et al. Brain-derived neurotrophic factor-deficient mice exhibit a hippocampal hyperserotonergic phenotype. Int J Neuropsychopharmacol 2008; 11(1): 79-92

28. Deltheil T, Guiard BP, Cerdan J et al. Behavioral and serotonergic consequences of decreasing or increasing hippocampus brain-derived neurotrophic factor protein levels in mice. Neuropharmacology 2008; 55(6): 1006-14

29. Nakagawasai O, Yamada K, Odaira T et al. Liver hydrolysate improves depressive-like behavior in olfactory bulbectomized mice: Involvement of hippocampal neurogenesis through the AMPK/BDNF/CREB pathway. J Pharmacol Sci 2020; 143(1): 52-5 


\section{Figure 1. Survival of newly generated cells and BDNF protein levels}

A. Treatment design. Mice received three injections of BrdU on day 1 followed by daily drug treatment for 21 days; SAL, saline, CIT, Citalopram, TIA, Tianeptine. B. Number of BrdU-positive cells in the dentate gyrus. CIT treatment increased the survival of BrdU-positive cells in CTR mice, while TIA had no effect. BrdU numbers in Tph2 ${ }^{-}$mice were significantly elevated relative to CTR mice in the SAL and TIA conditions, and without differences between treatment conditions. C. BDNF protein levels. In CTR mice, BDNF concentrations were not affected by treatment with either CIT or TIA. In comparison to CTR, BDNF protein levels in Tph2 ${ }^{-}$mice were significantly increased in the SAL and TIA conditions, and decreased following CIT. Two-way ANOVA followed by Tukey's post hoc test, ${ }^{*} p<0.05,{ }^{* *} p<0.01,{ }^{* * *} p<0.001$. D. Immunohistochemistry of cells co-labeled for BrdU/DCX (D1, arrow, scale bar $50 \mu \mathrm{m}$ ), BrdU/NeuN (D1, asterisk), BrdU/Sox2 (D2, arrow-head, scale bar $100 \mu \mathrm{m}$ ) and BrdU/CR (Calretinin, D2, asterisk). E-F. Quantitative assessment of newly generated cells. BrdU/Sox2 (precursor cells), BrdU/DCX (transient immature neurons), BrdU/CR (Calretinin, transient early post-mitotic neurons), BrdU/NeuN (mature granule cells). Phenotypes of BrdU-positive cells are represented as number (\#, E) and distribution (\%, F). [E, \#] Student's $t$ tests ${ }^{*} p<0.05,{ }^{* *} p<0.01,{ }^{* * *} p<0.001$. [F, \%] Two-way ANOVA followed by Tukey's post hoc test, ${ }^{*} p \leq 0.05 ; \# p<0.05$ individual comparison.

A

Treatment scheme

Day 0,1

Brdu fing on

B Adult neurogenesis

ㄷTR
Tph2-/-

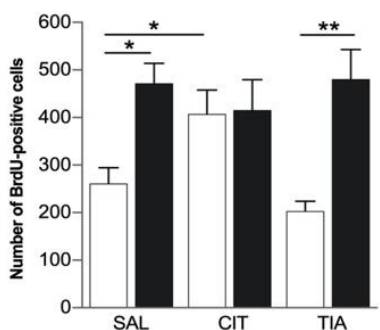

-

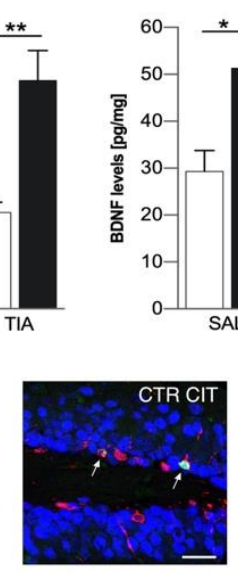

21 Dissected hippocampi 1. Adult neurogenesis 2. BDNF protein

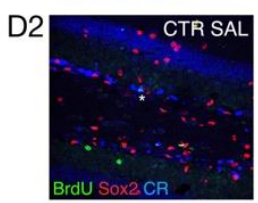

$\mathrm{D}$
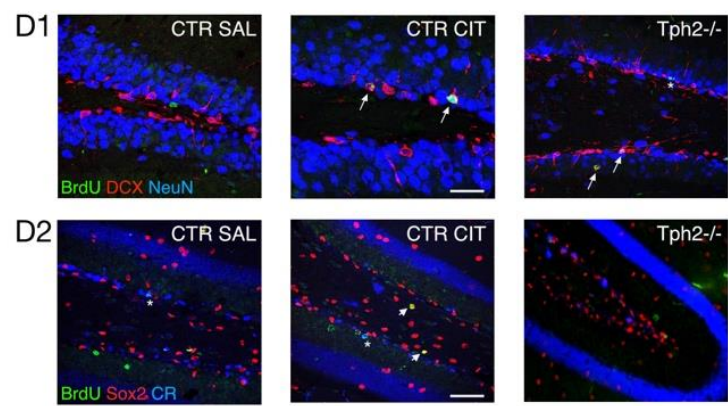

E

Phenotype analysis

口CTR

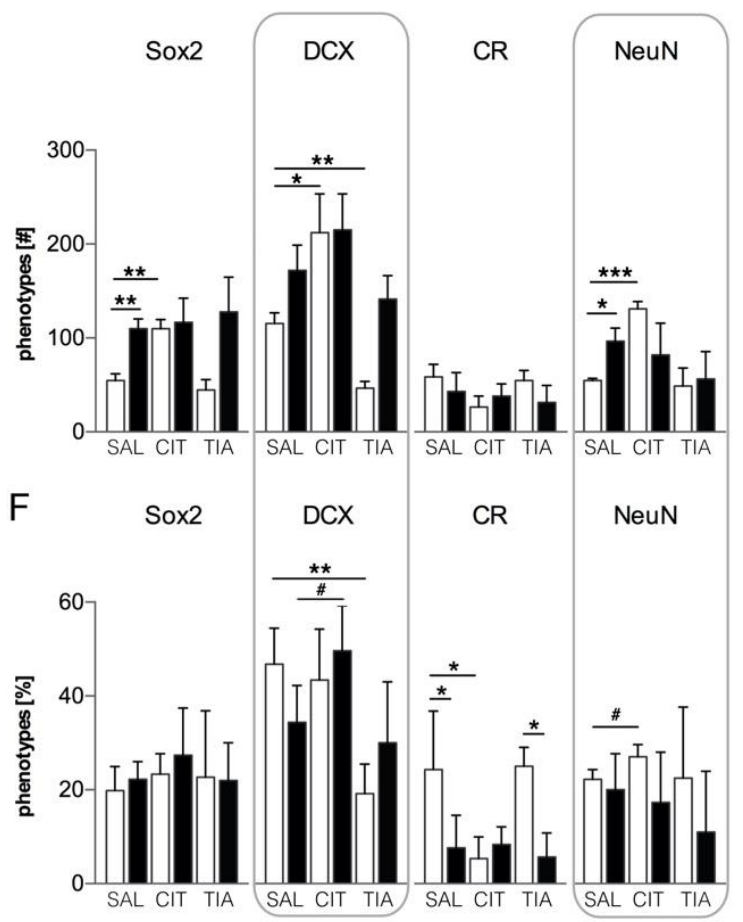

